\title{
Limit Cycles and Integrability in a Class of Systems with High-Order Nilpotent Critical Points
}

\author{
Feng Li and Jianlong Qiu \\ School of Science, Linyi University, Shandong, Linyi 276005, China \\ Correspondence should be addressed to Jianlong Qiu; qjllinyi@yahoo.com.cn
}

Received 17 December 2012; Accepted 2 February 2013

Academic Editor: Chuangxia Huang

Copyright ( 2013 F. Li and J. Qiu. This is an open access article distributed under the Creative Commons Attribution License, which permits unrestricted use, distribution, and reproduction in any medium, provided the original work is properly cited.

A class of polynomial differential systems with high-order nilpotent critical points are investigated in this paper. Those systems could be changed into systems with an element critical point. The center conditions and bifurcation of limit cycles could be obtained by classical methods. Finally, an example was given; with the help of computer algebra system MATHEMATICA, the first 5 Lyapunov constants are deduced. As a result, sufficient and necessary conditions in order to have a center are obtained. The fact that there exist 5 small amplitude limit cycles created from the high-order nilpotent critical point is also proved.

\section{Introduction}

In the qualitative theory of ordinary differential equations, bifurcation of limit cycles for planar polynomial differential systems which belong to second part of the Hilbert 16th problem is hot but intractable issue. Of course, this problem is far from being solved now; there are many famous works about this problem. Let $H(n)$ be the maximum possible number of limit cycles bifurcating from infinity for analytic vector fields of degree $n$. It was found that $N(3) \geq 7[1,2]$, $N(5) \geq 11[3], N(7) \geq 13$ [4].

When the critical point is a nilpotent critical point, let $N(n)$ be the maximum possible number of limit cycles bifurcating from nilpotent critical points for analytic vector fields of degree $n$. It was found that $N(3) \geq 2, N(5) \geq 5$, $N(7) \geq 9$ in [5], $N(3) \geq 3, N(5) \geq 5$ in [6], and for a family of Kukles systems with 6 parameters $N(3) \geq 3$ in [7]. Recently, li and liu other found that $N(3) \geq 8, N(5) \geq 12, N(7) \geq 13$ $[8,9]$ employing the inverse integral factor method.

But when the order of nilpotent critical point is higher than three, it is very difficult to study the limit cycle because the inverse integral factor method could not be used to compute the singular values. There are few results about the bifurcations of limit cycles at a nilpotent critical point with high order.
In this paper, we will study the bifurcation of limit cycles and conditions of centers for a class of special system

$$
\begin{aligned}
& \frac{d x}{d t}=y+\sum_{k=2}^{\infty} f_{k}\left(x^{n}, y\right), \\
& \frac{d y}{d t}=-x^{2 n-1}+x^{n-1} \sum_{k=2}^{\infty} g_{k}\left(x^{n}, y\right) .
\end{aligned}
$$

Obviously, when $n=1$, the system is

$$
\begin{aligned}
& \frac{d x}{d t}=y+\sum_{k=2}^{\infty} f_{k}(x, y), \\
& \frac{d y}{d t}=-x+\sum_{k=2}^{\infty} g_{k}(x, y) .
\end{aligned}
$$

The origin is an element critical point, but it is a high order nilpotent critical point when $n>1$.

This paper will be organized as follows. In Section 2, we state some preliminary knowledge given in [10] which is useful throughout the paper. In Section 3, we make some transformations to change system (2) into a system with an element singular. As an example, a special system is investigated. Using the linear recursive formulae in [10] to do 
direct computation, we obtain the first 5 Lyapunov constants and the sufficient and necessary conditions of center and the result that there exist 5 limit cycles in the neighborhood of the high-order nilpotent critical point are proved.

\section{Preliminary Knowledge}

The ideas of this section come from [10], where the center focus problem of critical points in the planar dynamical systems are studied. We first recall the related notions and results. For more details, please refer to [10].

In [10], the authors defined complex center and complex isochronous center for the following complex system:

$$
\begin{aligned}
& \frac{d z}{d T}=z+\sum_{k=2}^{\infty} \sum_{\alpha+\beta=k} a_{\alpha \beta} z^{\alpha} w^{\beta}=Z(z, w), \\
& \frac{d w}{d T}=-w-\sum_{k=2}^{\infty} \sum_{\alpha+\beta=k} b_{\alpha \beta} w^{\alpha} z^{\beta}=-W(z, w),
\end{aligned}
$$

and gave two recursive algorithms to determine necessary conditions for a center and an isochronous center. We now restate the definitions and algorithms.

Lemma 1. For system (3), we can derive uniquely the following formal series:

$$
\begin{aligned}
& \xi=z+\sum_{k+j=2}^{\infty} c_{k j} z^{k} w^{j}, \\
& \eta=w+\sum_{k+j=2}^{\infty} d_{k j} w^{k} z^{j},
\end{aligned}
$$

where $c_{k+1, k}=d_{k+1, k}=0, k=1,2, \ldots$, such that

$$
\begin{aligned}
& \frac{d \xi}{d T}=\xi+\sum_{j=1}^{\infty} p_{j} \xi^{j+1} \eta^{j}, \\
& \frac{d \eta}{d T}=-\eta-\sum_{j=1}^{\infty} q_{j} \eta^{j+1} \xi^{j} .
\end{aligned}
$$

Definition 2. Let $\mu_{0}=0, \mu_{k}=p_{k}-q_{k}, \tau_{k}=p_{k}+q_{k}, k=1,2, \ldots$. $\mu_{k}$ is called the $k_{\text {th }}$ singular point quantity of the origin of system (3) and $\tau_{k}$ is called the $k_{\text {th }}$ period constant of the origin of system (3).

Theorem 3. For system (3), the origin is a complex center if and only if $\mu_{k}=0, k=1,2, \ldots$. The origin is a complex isochronous center if and only if $\mu_{k}=\tau_{k}=0, k=1,2, \ldots$.

Theorem 4. For system (3), we can derive successively the terms of the following formal series:

$$
M(z, w)=\sum_{\alpha+\beta=0}^{\infty} c_{\alpha \beta} z^{\alpha} w^{\beta}
$$

such that

$$
\frac{\partial(M Z)}{\partial z}-\frac{\partial(M W)}{\partial w}=\sum_{m=1}^{\infty}(m+1) \mu_{m}(z w)^{m}
$$

where $c_{00}=1$, for all $c_{k k} \in R, k=1,2, \ldots$, and for any integer $m, \mu_{m}$ is determined by the following recursive formulae:

$$
\begin{aligned}
c_{\alpha \beta}= & \frac{1}{\beta-\alpha} \sum_{k+j=3}^{\alpha+\beta+2}\left[(\alpha+1) a_{k, j-1}\right. \\
& \left.-(\beta+1) b_{j, k-1}\right] c_{\alpha-k+1, \beta-j+1}, \\
\mu_{m}= & \sum_{k+j=3}^{2 m+2}\left(a_{k, j-1}-b_{j, k-1}\right) c_{m-k+1, m-j+1} .
\end{aligned}
$$

Theorem 5 (the constructive theorem of singular point quantities in [10]). A $k$-order singular point quantity of system (3) at the origin can be represented as a linear combination of $k$ order monomial Lie invariants and their antisymmetry forms, that is,

$$
\mu_{k}=\sum_{j=1}^{N} \gamma_{k j}\left(g_{k j}-g_{k j}^{*}\right), \quad k=1,2, \ldots
$$

where $N$ is a positive integer and $\gamma_{k j}$ is a rational number, $g_{k j}$ and $g_{k j}^{*}$ are $k$-order monomial Lie invariants of system (3).

Theorem 6 (the extended symmetric principle in [10]). Let $g$ denote an elementary Lie invariant of system (3). If for all $g$ the symmetric condition $g=g^{*}$ is satisfied, then the origin of system (3) is a complex center. Namely, all singular point quantities of the origin are zero.

\section{Simplification of System}

In fact, system (1) is symmetric with axis when $n$ is even, so the origin is a center. Now we will consider system (1) when $n=2 k+1$ is odd.

By transformations

$$
u=x^{2 k+1}, \quad v=y, \quad d \tau=u^{2 k /(2 k+1)} d t
$$

system (1) is changed into

$$
\begin{aligned}
& \frac{d u}{d \tau}=(2 k+1) v+(2 k+1) \sum_{k=2}^{\infty} f_{k}(u, v), \\
& \frac{d v}{d \tau}=-u+\sum_{k=2}^{\infty} g_{k}(u, v) .
\end{aligned}
$$

then by transformations

$$
x=u, \quad y=\sqrt{2 k+1} v, \quad d \tau=\sqrt{2 k+1} d t,
$$

system (11) could be changed into

$$
\begin{aligned}
& \frac{d x}{d \tau}=-y-\sqrt{2 k+1} \sum_{k=2}^{\infty} f_{k}\left(x, \frac{1}{\sqrt{2 k+1}} y\right), \\
& \frac{d v}{d \tau}=x-\sum_{k=2}^{\infty} g_{k}\left(x, \frac{1}{\sqrt{2 k+1}} y\right) .
\end{aligned}
$$


Thus, the origin of system (13) is an element critical point. It could be investigated using the classical integral factor method.

Now, we consider the following system:

$$
\begin{aligned}
& \frac{d x}{d t}=y+A_{30} x^{3 n}+A_{21} x^{2 n} y+A_{12} x^{n} y^{2}+A_{03} y^{3}, \\
& \frac{d y}{d t}=-x^{2 n-1}+x^{n-1}\left(B_{30} x^{3 n}+B_{21} x^{2 n} y+B_{12} x^{n} y^{2}+B_{03} y^{3}\right) .
\end{aligned}
$$

When $n=2 k+1$, by those transformations, system (14) is changed into

$$
\begin{gathered}
\frac{d x}{d t}=-y-\sqrt{2 k+1}\left(A_{30} x^{3}+\frac{A_{21}}{\sqrt{2 k+1}} x^{2} y\right. \\
\left.+\frac{A_{12}}{2 k+1} x y^{2}+\frac{A_{03}}{(2 k+1)^{3 / 2}}\right), \\
\frac{d y}{d t}=x-\left(B_{30} x^{3}+\frac{B_{21}}{\sqrt{2 k+1}} x^{2} y+\frac{B_{12}}{2 k+1} x y^{2}\right. \\
\left.+\frac{B_{03}}{(2 k+1)^{3 / 2}} y^{3}\right)
\end{gathered}
$$

where

$$
\begin{gathered}
A_{30}=\frac{-2 A_{2}-2 A_{3}-3 A_{4}+2 A_{2} k+A_{4} k}{16(1+2 k)^{3}}, \\
A_{21}=\frac{-9 B_{1}+B_{2}+B_{3}+3 B_{1} k}{8(1+2 k)^{5 / 2}} \\
A_{12}=\frac{-2 A_{2}-2 A_{3}+9 A_{4}+2 A_{2} k-3 A_{4} k}{16(1+2 k)^{2}}, \\
A_{03}=\frac{3 B_{1}+B_{2}+B_{3}-B_{1} k}{8(1+2 k)^{3 / 2}} \\
B_{21}=\frac{-2 A_{2}+2 A_{3}-45 A_{4}+2 A_{2} k+15 A_{4} k}{16(1+2 k)^{2}}, \\
B_{30}=\frac{3 B_{1}-B_{2}+B_{3}-B_{1} k}{8(1+2 k)^{5 / 2}} \\
B_{12}=\frac{-9 B_{1}-B_{2}+B_{3}+3 B_{1} k}{8(1+2 k)^{3 / 2}}, \\
-2 A_{2}+2 A_{3}+15 A_{4}+2 A_{2} k-5 A_{4} k \\
16(1+2 k)
\end{gathered}
$$

By transformation

$$
z=x+i y, \quad w=x-i y, \quad T=i t,
$$

system (15) is changed into

$$
\begin{aligned}
& \frac{d z}{d T}=z+a_{30} z^{3}+a_{21} z^{2} w+a_{12} z w^{2}+a_{03} w^{3}, \\
& \frac{d w}{d T}=-w-b_{30} w^{3}-b_{21} w^{2} z-b_{12} w z^{2}-b_{03} z^{3},
\end{aligned}
$$

where

$$
\begin{gathered}
a_{30}=\frac{\left(3 i A_{4}+2 B_{1}\right)(k-3)}{16(1+2 k)^{5 / 2}}, \\
a_{21}=\frac{-i A_{2}+B_{2}+i A_{2} k}{8(1+2 k)^{5 / 2}}, \\
a_{12}=-\frac{i\left(A_{3}-i B_{3}\right)}{8(1+2 k)^{5 / 2}}, \\
a_{03}=-\frac{i A_{4}(k-3)}{8(1+2 k)^{5 / 2}}, \quad b_{i j}=\overline{a_{i j}} .
\end{gathered}
$$

After careful computation by using formula in (4), we have the following.

Theorem 7. For system (18), the first 5 Lyapunov constants at the origin are given by

$$
\begin{aligned}
& \lambda_{1}=\frac{i A_{2}(k-1)}{4(1+2 k)^{5 / 2}}, \\
& \lambda_{2}=\frac{i\left(2 A_{3} B_{1}+3 A_{4} B_{3}\right)(k-3)}{64(1+2 k)^{5}} .
\end{aligned}
$$

When $A_{4} B_{1} \neq 0$

$$
\begin{aligned}
& \lambda_{3}=\frac{i A_{4}\left(3 A_{4}-2 B_{1}\right)\left(3 A_{4}+2 B_{1}\right)(k-3)(-9+3 k-2 p)(-3+k+6 p)}{8192(1+2 k)^{15 / 2}}, \\
& \lambda_{4}=\frac{i A_{4} B_{2}\left(3 A_{4}-2 B_{1}\right)\left(3 A_{4}+2 B_{1}\right)(k-3)^{2}(-9+3 k-2 p)}{49152(1+2 k)^{10}}, \\
& \lambda_{5}=\frac{i A_{4} B_{1}^{2}\left(3 A_{4}-2 B_{1}\right)\left(3 A_{4}+2 B_{1}\right)(k-3)^{4}(-9+3 k-2 p)}{2654208(1+2 k)^{25 / 2}} .
\end{aligned}
$$

When $A_{4}=0, B_{1} \neq 0$

$$
\lambda_{2}=\frac{i A_{3} B_{1}(k-3)}{32(1+2 k)^{5}} .
$$

When $A_{4} \neq 0, B_{1}=0$

$$
\begin{aligned}
& \lambda_{2}=\frac{3 i A_{4} B_{3}(k-3)}{64(1+2 k)^{5}}, \\
& \lambda_{3}=\frac{3 i A_{4}\left(2 A_{3}-3 A_{4}+A_{4} k\right)\left(-2 A_{3}-27 A_{4}+9 A_{4} k\right)(k-3)}{8192(1+2 k)^{15 / 2}}, \\
& \lambda_{4}=-\frac{i A_{4} B_{2}\left(-2 A_{3}-27 A_{4}+9 A_{4} k\right)(k-3)^{2}}{49152(1+2 k)^{10}} .
\end{aligned}
$$

When $A_{4}=B_{1}=0$

$$
\lambda_{2}=\lambda_{3}=\lambda_{4}=\cdots=0 .
$$

In the above expression of $\lambda_{k}$, one has already let $\lambda_{1}=\lambda_{2}=$ $\lambda_{3}=\lambda_{4}=0$. 
From Theorem 7, we obtain the following assertion.

Proposition 8. The first 5 Lyapunov constants at the origin of system (18) are zero if and only if one of the following conditions is satisfied:

$$
\begin{aligned}
& k=3, \quad A_{2}=0, \\
& A_{2}=0, \quad A_{3}=\frac{3(3 k-9)}{2} A_{4}, \quad B_{3}=-2(3 k-9) B_{1} \text {, } \\
& A_{2}=0, \quad 2 A_{3} B_{1}=-3 A_{4} B_{3}, \quad B_{1}=-\frac{3}{2} A_{4}, \\
& A_{2}=0, \quad 2 A_{3} B_{1}=-3 A_{4} B_{3}, \quad B_{1}=\frac{3}{2} A_{4}, \\
& A_{2}=A_{3}=A_{4}=0 \text {, } \\
& A_{2}=B_{1}=B_{2}=B_{3}=0, \quad A_{3}=-\frac{k-3}{2} A_{4} \text {, } \\
& k=1, \quad A_{3}=-9 A_{4}, \quad B_{3}=6 B_{1} \text {, } \\
& k=1, \quad 2 A_{3} B_{1}=-3 A_{4} B_{3}, \quad B_{1}=-\frac{3}{2} A_{4}, \\
& k=1, \quad 2 A_{3} B_{1}=-3 A_{4} B_{3}, \quad B_{1}=\frac{3}{2} A_{4}, \\
& k=1, \quad A_{3}=A_{4}=0, \\
& k=1, \quad B_{1}=B_{2}=B_{3}=0, \quad A_{3}=A_{4} \text {. }
\end{aligned}
$$

Furthermore, we have the following.

Theorem 9. The origin of system (18) is a center if and only if the first 5 Lyapunov constants are zero; that is, one of the conditions in Proposition 8 is satisfied.

Proof. When one of conditions (25), (27), (28), (29), (30), (32), (33), and (34) holds, according to Theorems 6, we get all $\mu_{k}=0, k=1,2 \ldots$

When condition (26) holds, system (18) could be written as

$$
\begin{aligned}
\frac{d x}{d t}= & -y+\frac{(k-3) A_{4}}{2(1+2 k)^{5 / 2}} x^{3}-\frac{B_{2}}{8(1+2 k)^{5 / 2}} x^{2} y \\
& +\frac{3(k-3) A_{4}}{4(1+2 k)^{5 / 2}} x y^{2}+\frac{4 B_{1} k-B_{2}-12 B_{1}}{8(1+2 k)^{5 / 2}} y^{3}, \\
\frac{d y}{d t}= & x+\frac{4 B_{1} k+B_{2}-12 B_{1}}{8(1+2 k)^{5 / 2}} x^{3}-\frac{3(k-3) A_{4}}{2(1+2 k)^{5 / 2}} x^{2} y \\
& +\frac{B_{2}}{8(1+2 k)^{5 / 2}} x y^{2}-\frac{(n-3) A_{4}}{4(1+2 k)^{5 / 2}} y^{3} .
\end{aligned}
$$

System (36) has an analytic first integral

$$
\begin{aligned}
H(x, y)= & \frac{1}{2} x^{2}+\frac{1}{2} y^{2}+\frac{4 B_{1} n+B_{2}-12 B_{1}}{32(1+2 k)^{5 / 2}} x^{4} \\
& -\frac{(k-3) A_{4}}{2(1+2 k)^{5 / 2}} x^{3} y+\frac{B_{2}}{16(1+2 k)^{5 / 2}} x^{2} y^{2} \\
& -\frac{k-3}{4(1+2 k)^{5 / 2}} A_{4} x y^{3}-\frac{4 B_{1} n-B_{2}-12 B_{1}}{32(1+2 k)^{5 / 2}} y^{4}
\end{aligned}
$$

When condition (31) holds, system (18) could be written as

$$
\begin{aligned}
\frac{d x}{d t}=-y+\sqrt{3}\left(\frac{A_{4}}{27} x^{3}-\frac{B_{2}}{216} x^{2} y\right. & \\
& \left.+\frac{A_{4}}{18} x y^{2}-\frac{-8 B_{1}+B_{2}}{216} y^{3}\right), \\
\frac{d y}{d t}= & x+\frac{-8 B_{1}+B_{2}}{72 \sqrt{3}} x^{3}+\frac{A_{4}}{3 \sqrt{3}} x^{2} y \\
+ & \frac{B_{2}}{72 \sqrt{3}} x y^{2}+\frac{A_{4}}{18 \sqrt{3}} y^{3} .
\end{aligned}
$$

System (38) has an analytic first integral

$$
\begin{aligned}
H(x, y)= & \frac{1}{2} x^{2}+\frac{1}{2} y^{2}-\frac{-8 B_{1}-B_{2}}{864} y^{4}+\frac{\sqrt{3}\left(-8 B_{1}+B_{2}\right)}{864} x^{4} \\
& +\frac{\sqrt{3}}{432} B_{2} x^{2} y^{2}+\frac{\sqrt{3} A_{4}}{9} x^{3} y .
\end{aligned}
$$

When condition (35) holds, system (18) could be written as

$$
\begin{aligned}
& \frac{d x}{d t}=-y+\frac{\sqrt{3} A_{4}}{108} x^{3}-\frac{\sqrt{3} A_{4}}{108} x y^{2}, \\
& \frac{d y}{d t}=x+\frac{7 \sqrt{3} A_{4}}{108} x^{2} y-\frac{\sqrt{3} A_{4}}{36} y^{3} .
\end{aligned}
$$

System (40) has an analytic first integral

$$
H(x, y)=\frac{1}{2} x^{2}+\frac{1}{2} y^{2}-\frac{\sqrt{3} A_{4}}{108} x^{3} y-\frac{\sqrt{3} A_{4}}{36} x y^{3} .
$$

Next, we will prove that when the critical point $O(0,0)$ is a 5 -order weak focus, the perturbed system of (15) can generate 5 limit cycles enclosing the origin of perturbation system (15).

Using the fact

$$
\lambda_{1}=\lambda_{2}=\lambda_{3}=\lambda_{4}=0, \quad \lambda_{5} \neq 0,
$$

we obtain the following. 
Theorem 10. The origin of system (18) is a 5-order weak focus if and only if one of the following conditions is satisfied:

$$
\begin{gathered}
A_{2}=0, \quad A_{3}=\frac{3-k}{2} A_{4}, \quad B_{3}=-\frac{3-k}{3} B_{1}, \quad B_{2}=0, \\
A_{4} B_{1}^{2}\left(3 A_{4}-2 B_{1}\right)\left(3 A_{4}+2 B_{1}\right)(k-3) \neq 0, \\
k=1, \quad A_{3}=A_{4}, \quad B_{3}=-\frac{2}{3} B_{1}, \quad B_{2}=0, \\
A_{4} B_{1}\left(3 A_{4}-2 B_{1}\right)\left(3 A_{4}+2 B_{1}\right) \neq 0 .
\end{gathered}
$$

We next study the perturbed system of (15) as follows:

$$
\begin{gathered}
\frac{d x}{d t}=\delta x-y-\sqrt{2 k+1}\left(A_{30} x^{3}+\frac{A_{21}}{\sqrt{2 k+1}} x^{2} y\right. \\
\left.+\frac{A_{12}}{2 k+1} x y^{2}+\frac{A_{03}}{(2 k+1)^{3 / 2}}\right), \\
\frac{d y}{d t}=\delta y+x-\left(B_{30} x^{3}+\frac{B_{21}}{\sqrt{2 k+1}} x^{2} y\right. \\
\left.+\frac{B_{12}}{2 k+1} x y^{2}+\frac{B_{03}}{(2 k+1)^{3 / 2}}\right)
\end{gathered}
$$

Theorem 11. If the origin of system (15) is a 5-order weak focus, for $0<\delta \ll 1$, making a small perturbation to the coefficients of system (15), then, for system (45), in a small neighborhood of the origin, there exist exactly 5 small amplitude limit cycles enclosing the origin $\mathrm{O}(0,0)$.

Proof. It is easy to check that when condition (43) or (44) holds,

$$
\frac{\partial\left(\lambda_{1}, \lambda_{2}, \lambda_{3}, \lambda_{4}\right)}{\partial\left(A_{2}, A_{3}, B_{3}, B_{2}\right)} \neq 0 .
$$

From the statement mentioned above, according to the classical theory of Bautin, there exist 5 limit cycles in a small enough neighborhood of the origin.

\section{Acknowledgments}

This research is partially supported by the National Nature Science Foundation of China (11201211, 61273012) and Project of Shandong Province Higher Educational Science and Technology Program (J12LI58).

\section{References}

[1] Q. Zhang and Y. Liu, "A cubic polynomial system with seven limit cycles at infinity," Applied Mathematics and Computation, vol. 177, no. 1, pp. 319-329, 2006.

[2] Y. Liu and W. Huang, "Seven large-amplitude limit cycles in a cubic polynomial system," International Journal of Bifurcation and Chaos, vol. 16, no. 2, pp. 473-485, 2006.
[3] Q. Zhang and Y. Liu, "A quintic polynomial differential system with eleven limit cycles at the infinity," Computers \& Mathematics with Applications, vol. 53, no. 10, pp. 1518-1526, 2007.

[4] Q. Zhang, G. Weihua, and Y. Liu, "Bifurcation of limit cycles at the equator for a class of polynomial differential system," Nonlinear Analysis: Real World Applications, vol. 10, no. 2, pp. 1042-1047, 2009.

[5] A. F. Andreev, A. P. Sadovskiŭ, and V. A. Tsikalyuk, "The centerfocus problem for a system with homogeneous nonlinearities in the case of zero eigenvalues of the linear pact," Differential Equations, vol. 39, no. 2, pp. 155-164, 2003.

[6] M. J. Álvarez and A. Gasull, "Monodromy and stability for nilpotent critical points," International Journal of Bifurcation and Chaos, vol. 15, no. 4, pp. 1253-1265, 2005.

[7] M. J. Álvarez and A. Gasull, "Generating limit cycles from a nilpotent critical point via normal forms," Journal of Mathematical Analysis and Applications, vol. 318, no. 1, pp. 271-287, 2006.

[8] F. Li, "Bifurcations of limit cycles in a quintic Lyapunov system with eleven parameters," Chaos, Solitons \& Fractals, vol. 45, no. 11, pp. 1417-1422, 2012.

[9] F. Li, Y. Liu, and H. Li, "Center conditions and bifurcation of limit cycles at three-order nilpotent critical point in a septic Lyapunov system," Mathematics and Computers in Simulation, vol. 81, no. 12, pp. 2595-2607, 2011.

[10] Y. Liu and J. Li, Some Classical Problems about Planar Vector Fields, Science Press, Beijing, China, 2010. 


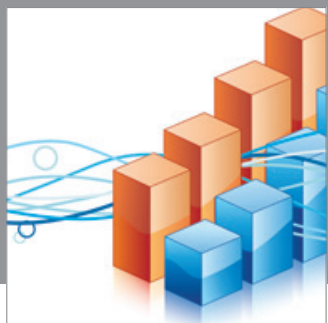

Advances in

Operations Research

mansans

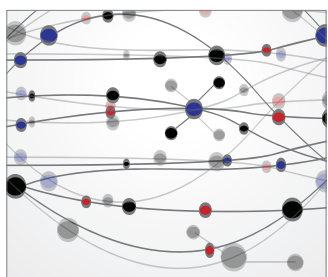

The Scientific World Journal
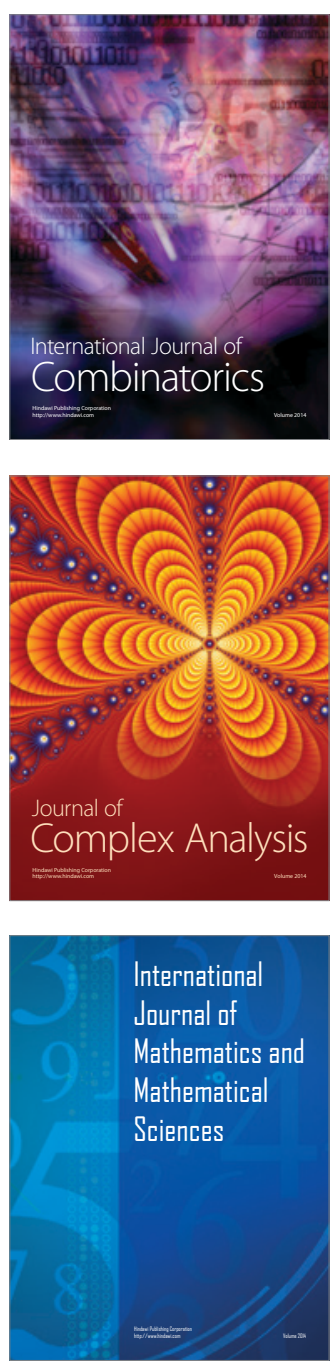
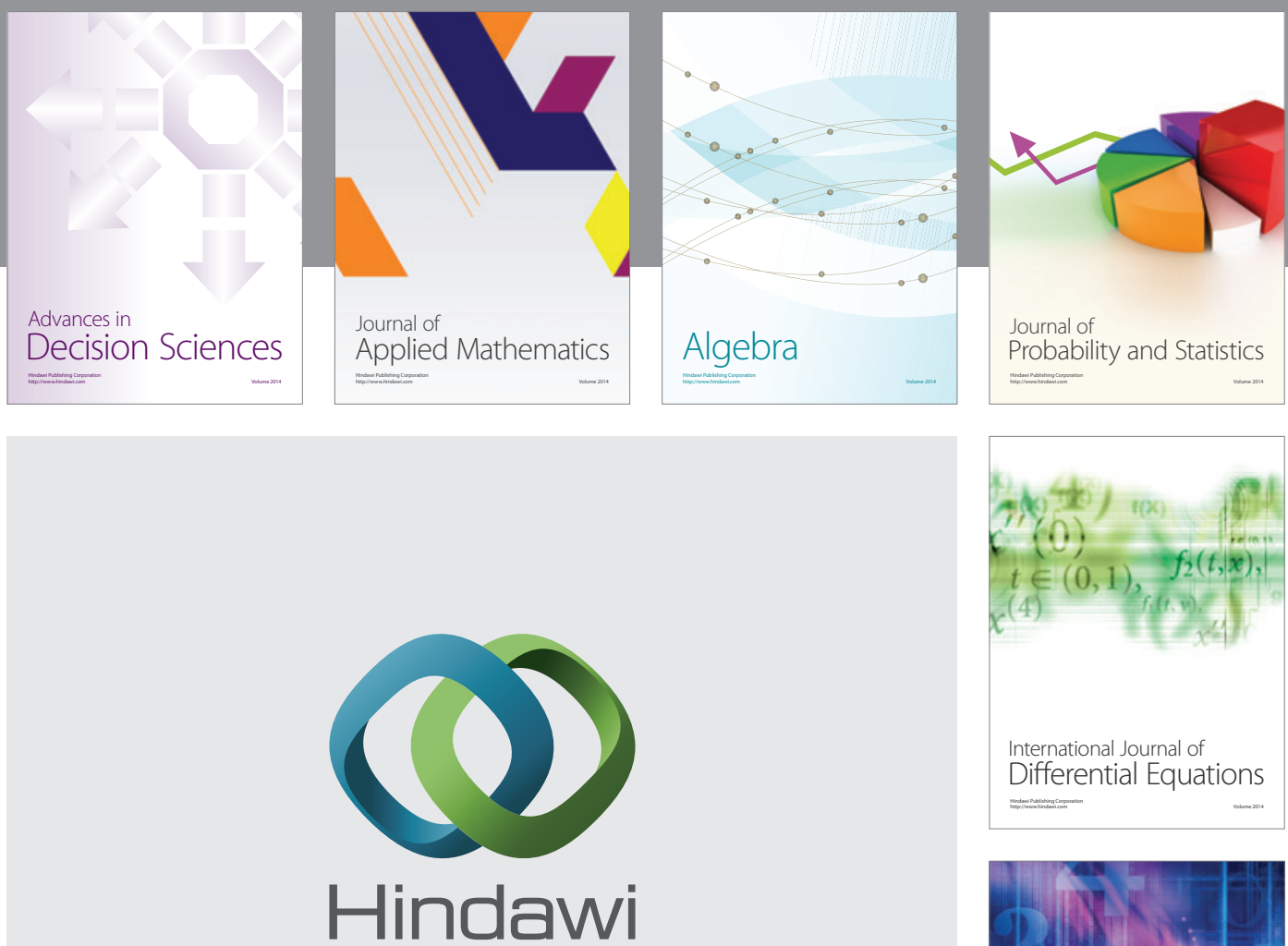

Submit your manuscripts at http://www.hindawi.com
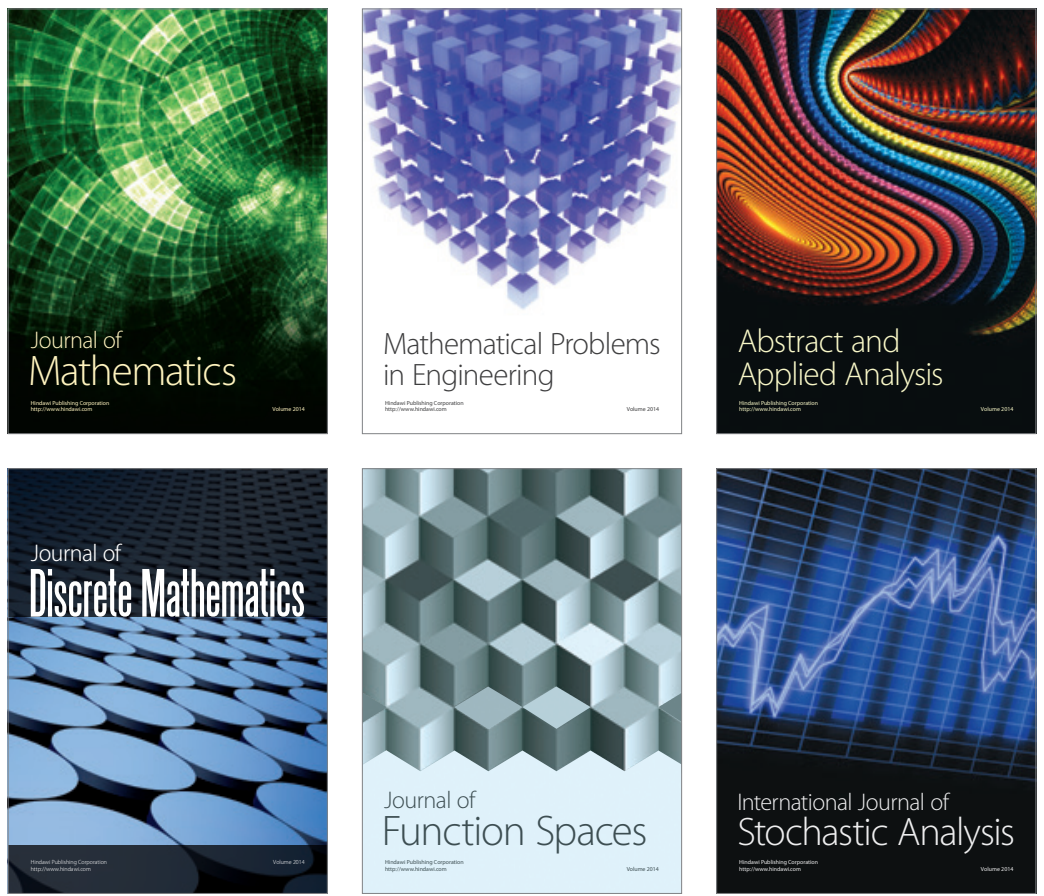

Journal of

Function Spaces

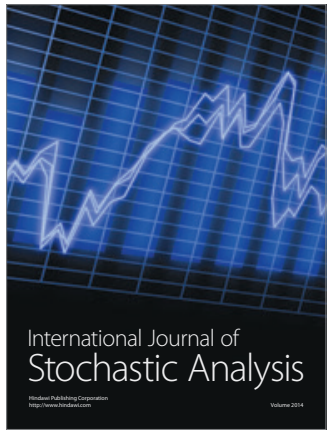

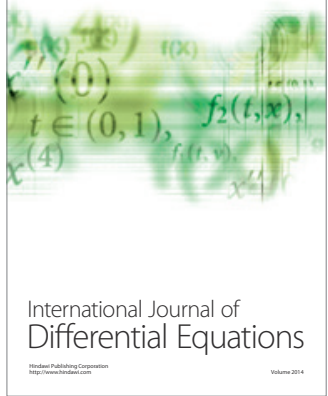
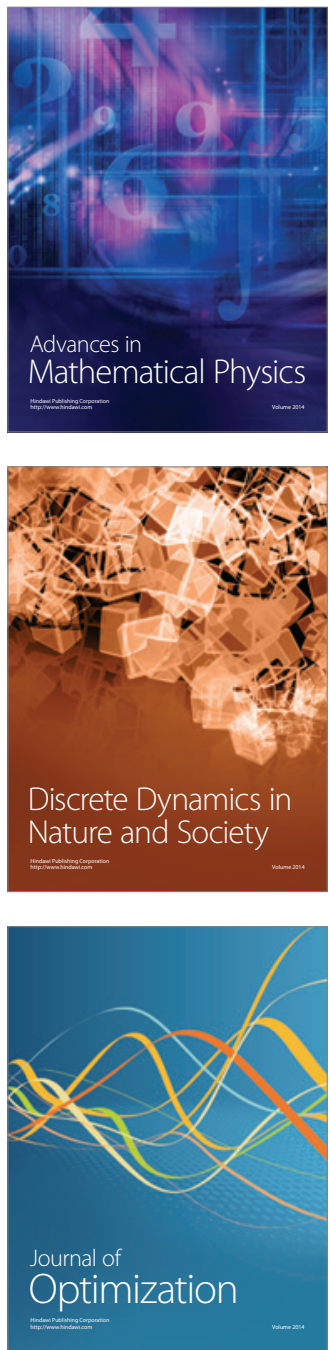